\title{
What is wrong with compliance?
}

\author{
Søren Holm Institute of Biostatistics and Theory of Medicine, Copenhagen, Denmark
}

\section{Author's abstract}

Non-compliance is a label often used about patients who do not follow therapeutic advice. This paper analyses the notion of compliance, and tries to show that this notion is inextricably bound to a paternalistic conception of the doctor-patient relationship. It is proposed that we should perhaps not talk so much about the non-compliant patient, but instead shift the focus towards the noncompliant doctor.

'To comply: To act in accordance with, and fulfilment of, wishes, desires, requests, demands, conditions or regulations; to fulfil the wishes or requirements of ; to yield, accede, or consent to.'

'Compliant: Complying, disposed to comply. Ready to yield to the wishes or desires of others.' (1).

'Non-compliance: Failure or refusal to comply'

Every doctor can tell stories about the diabetic who 'cheats', the epileptic who, without consulting the doctor, stops taking his medication, or the patient who 'misuses' her steroid cream. All these patients will, in common parlance, be labelled as non-compliant, and medical students are often warned about this troublesome class of patients. But what is seldom realised is that this label is extremely value-laden, and points towards one specific highly paternalistic conception of the doctor-patient relationship.

\section{The meaning of non-compliance}

It has no meaning to talk about a person who is noncompliant with respect to herself. If I do not follow my own wishes or desires, I may be fainthearted or weak-willed, but I am not non-compliant. It therefore follows, that if the non-compliant patient is the patient who fails or refuses to comply, it must be because she fails or refuses to act in accordance with somebody else's wishes, desires, requests, demands, conditions or regulations. She fails or refuses to yield

\section{Key words}

Compliance; patients' rights. to somebody else's will. And in the case of a person in the patient role, this somebody else can only be the doctor (or some other health care professional).

This clearly points to a radically paternalistic conception of the doctor-patient relationship, where the doctor decides and the patient complies. Watkins has described two mutually exclusive views of the role of the doctor as expert, and the use of terms like compliance clearly indicate an acceptance of the view that:

'... experts [are] people with autonomous power over others, based upon incomprehensible and exclusive bodies of knowledge, who derive status from the power and mystique of their position' (2).

As we (slowly) move away from the paternalistic conception of the doctor-patient relationship, to a form of relationship where the patient's autonomy and fundamental right to self-determination is acknowledged, we should also abandon the present conception of compliance. If it is ultimately the patient who has to decide, after being duly informed and advised, then he cannot be non-compliant. $\mathrm{He}$ may be non-collaborative, obstructive, foolish or stupid if he blatantly disregards the decisions to which he is a party, but since they are his decisions and not the doctor's orders this does not imply noncompliance. And there may in fact be excellent reasons for changing the treatment-plan which was originally agreed (see below). But old manners are hard to eradicate. In a recent article about the duties of the patient vis-a-vis the doctor we read:

'One very obvious duty is that to collaborate, from which can be derived ..., an obligation to follow therapeutic advice, ie a duty of compliance ...' (3).

If such a duty of compliance exists, it cannot be derived from a duty to collaborate. Collaboration describes a situation where two or more partners are involved in a common endeavour. Whereas compliance only has a place if one partner is totally in control. It makes no sense talking about a duty to follow (or comply with) therapeutic advice, for such a duty would immediately transform advice to 
'doctor's orders'. Advice is only advice if it can be rejected. If there is a duty to collaborate with the doctor, then it must be understood as a duty to collaborate in the diagnostic process until sufficient information has been gathered to make a treatment decision, at which point the patient is free to make his own decision.

Within the paternalistic framework it is commonly presupposed, that the compliant patient is the 'good' patient, and the non-compliant the 'bad' or troublesome patient. The non-compliant patient is seen as not fulfilling her duty towards the doctor, and therefore as morally culpable. But even on its own terms this attitude runs into problems, because there seems to be no good way of grading the moral import of non-compliance. To take an example: Who is most to blame, the person whose previous lifestyle and liking of vegetables and exercise fits well with his diabetic regime but who is careless with his insulin, or the person whose dream of the good life is a box of chocolates, a good book, and an armchair by the fire and who fights his natural inclinations but never quite succeeds. They may both have bad control, but who is most to blame?

The attitude that non-compliance implies moral culpability is, however, reinforced by typologies of non-compliant behaviour which do not admit that there can be very good reasons for non-compliance:

'Compliance can be viewed as both an attitude and a behaviour. Compliance as an attitude consists of a willingness or intention to follow health prescriptions, whereas behaviour relates to the actual carrying out of prescriptions. Non-compliant behaviour could then include reluctance, reactance, and recidivism characterised by disinterest, refusal to comply, or the lack of sustained effort to follow health recommendations' (4).

If doctors never made mistakes, were never insensitive to their patients' requests, and never prescribed any treatment without adequate information of, and discussion with, the patient, then it might conceivably be true that departures from the prescribed treatment could be exhaustively explained as caused by lack of interest, refusal to comply, or the lack of sustained effort on the part of the patient. And in that situation we might claim that the patient was to some degree morally culpable if the departure from treatment had untoward consequences.

But we know that the system does not work that way. Doctors often prescribe 'standard' treatments from the book without sufficient regard to the patient's specific situation, and without sufficient discussion. And in that situation the patient's departures from the prescribed treatment may represent a fully rational decision and be the best compromise between the patient's long term life-plan and his disease.

These kinds of problems are especially vivid in the case of the patient with chronic disease requiring continuous medication (for example epilepsy or diabetes). In these cases the doctor's claim to special knowledge and expertise, which normally supports the doctor's right to be the decision-maker instead of the patient, is severely weakened. It is obvious that doctors in general will be more knowledgeable than the average patient with a fracture, an abscess or a bout of pneumonia, but chronic diseases involve the patient in a quite different way. The patients quickly learn the specific features of the disease as it manifests itself in them. And whereas the doctor may be an expert on the disease in general, the patient soon becomes the foremost expert on her disease and its specific manifestations. The patient may know that strict adherence to the regime prescribed by the doctor will lead to severe and incapacitating episodes of side-effects, and it seems ludicrous to speak of 'non-compliance' if the patient alters the regime to gain a tolerable life. We do not, after all, treat diabetics to keep their glucose or $\mathrm{HgbA}_{1 \mathrm{c}}$ levels low, or epileptics to keep a steady serum phenantoin level, but to enable them to have a long and good life.

\section{Conclusion}

The intrinsic value judgement in the label 'noncompliant' makes it, as shown above, totally unsuitable for use in present-day medicine. If patients are to be involved as equal (or maybe even senior) partners in decisions concerning their health care, then doctors will have to adjust their role from being the sole decision-makers to being the expert advisers. Continued use of words like 'compliant' and 'non-compliant', which directly point to a quite different role for the doctor can only hinder this process of change.

It is a sign of hope that Index Medicus has no entry under the term 'compliance' but instead talks about 'co-operative behaviour', and that the terminology of 'adherence to treatment' is slowly gaining ground. But new terminology will not be sufficient, a change of the underlying attitudes is absolutely necessary.

It is not patients who should comply with their doctors' demands, but doctors who should comply with their patients' informed and considered desires. Maybe the time has now come to talk about the problems caused by the non-compliant doctor, instead of the non-compliant patient!

Soren Holm is a physician working in Copenhagen, Denmark. He holds an MA in Health Care Ethics from the Centre for Social Ethics and Policy, University of Manchester.

\section{References}

(1) The Oxford English Dictionary. Oxford: Clarendon Press, 1989.

(2) Watkins S. Medicine and labour - the politics of a profession. Lawrence and Wishart: London, 1987: 161. 
(3) Osinga M. But the patient has responsibilities as well! Fournal of the Medical Defence Union 1989; 5: 55-56.

(4) Schlenk E A, Hart L K. Relationship between health locus of control, health value, and social support and compliance of persons with diabetes mellitus. Diabetes care 1984; 7: 566-574. 